\title{
ASCO virtual meeting 2020: highlights head and neck cancer
}

\author{
Thorsten Fuereder (iD)
}

Received: 25 October 2020 / Accepted: 10 January 2021 / Published online: 27 January 2021

(C) The Author(s) 2021

\begin{abstract}
Summary At the ASCO 2020 virtual meeting, multiple clinically relevant studies were presented addressing open questions in the head and neck cancer field: Are de-escalation strategies feasible in low risk patients? What is the appropriate platinum dose in combination with radiotherapy in high-risk patients suffering from locally advanced disease? Is immunotherapy the first line standard of care for all patient in the recurrent/metastatic setting? This article summarizes the most significant head and neck cancer studies presented at the ASCO 2020 virtual meeting and discusses the data in the context of the current literature.
\end{abstract}

Keywords Recurrent/metastatic HNSCC · Locally advanced HNSCC · Immunotherapy · Radiotherapy • Surgery

\section{Introduction}

Head and neck cancer (HNSCC) data presented at this year's virtual American Society of Clinical Oncology (ASCO) scientific meeting were not entirely practice changing, although a couple of open questions were clarified and/or the current evidence confirmed. The most relevant clinical trials for our daily clinical practice included studies in early and locally advanced HNSCC. In the recurrent/metastatic (R/M) setting the focus was laid on immunotherapy studies. Since the magnitude of clinical benefit from new therapeutic approaches is often biased and a matter of debate [1], the author rated the outcome of the presented phase III studies according to the magnitude of clini-

\section{T. Fuereder, MD ( $\varangle)$}

Department of Medicine I and Comprehensive Cancer Center, Division of Clinical Oncology, Medical University of Vienna, Währinger Gürtel 18-20, 1090 Vienna, Austria thorsten.fuereder@meduniwien.ac.at cal benefit scale (MCBS) as suggested by the European Society of Medical Oncology (ESMO) [2], when applicable.

\section{Resectable early and locally advanced HNSCC}

\section{Senti-Mer study: sentinel lymph node biopsy vs.} elective neck dissection [3]

This multicenter randomized equivalence phase III study included 307 patients with stage T1-2 oral (OSCC) and oropharyngeal carcinoma (OPC) without clinical lymph node metastasis (N0). Patients were randomized to a standard of care (SOC) arm receiving elective neck dissection and to an experimental group undergoing sentinel lymph node biopsy. In case of positivity an elective neck dissection was performed as well, otherwise the patients were subjected to observation. The primary endpoint was 2-year neck node recurrence-free survival (RFS) and was met: The neck node RFS was comparable between the SOC group (89.6\%; 95\% confidence interval [CI] 0.83-0.94) and the experimental arm (89.4\%; 95\% CI 0.82-0.94). The locoregional RFS $(73.6 \%$; $95 \%$ CI $0.64-0.81$ vs. 76.3\%; 95\% CI 0.67-0.83) and overall survival (OS) (81.8\%; 95\% CI $0.74-0.88$ vs. $82.3 \%$; 95\% CI $0.74-0.88$ ) were similar between the two treatment arms. Morbidity endpoints, however, favored the sentinel lymph node group: Morbidity such as shoulder stiffness, arm abduction and ability to reach above one's head was significantly lower in the experimental group. Additionally, the need for physiotherapy was lower and the hospital stay shorter. The ESMO MCBS as rated by the author is $B$, i.e., relevant clinical benefit in the curative setting (non-inferior RFS/OS, but improved toxicity compared to standard of care). 


\section{E3311 study: transoral robotic surgical resection} followed by low- or standard dose radiotherapy [4]

The E3311 phase II de-escalation study evaluated the efficacy of risk-adjusted postoperative radiotherapy (50 Gy in 25 fractions vs. $60 \mathrm{~Gy}$ in 30 fractions) in p16 positive T1-2, N1-N2b OPC patients amenable to transoral robotic surgical resection. Primary endpoint was progression-free survival (PFS). Patients in the intermediate risk group defined as close margins (<3 mm), 2-4 positive lymph nodes, extracapsular lymph node extension $(<1 \mathrm{~mm})$ or perineural invasion were randomized to risk-adjusted postoperative radiotherapy. Low-risk patients entered follow-up and high-risk patients received chemoradiation. The PFS rate, however, for intermediate risk patients receiving low-dose radiation ( $n=102)$ was comparable with the SOC arm $(n=104)$ (PFS: 95.0\%; 90\% CI 91.4-98.6 vs. 95.9\%; 90\% CI 92.6-99.3).

\section{Adjuvant radiochemotherapy with bolus cisplatin vs. weekly cisplatin [5]}

The randomized Japanese JCOG1008 phase II/III study aimed at demonstrating the noninferiority of weekly $40 \mathrm{mg} / \mathrm{m}^{2}$ cisplatin to conventional SOC highdose $100 \mathrm{mg} / \mathrm{m}^{2}$ cisplatin administered every 3 weeks in stage III/IV HNSCC patients, who were treated with postoperative radiotherapy due to positive margins or extracapsular extension. OS was defined as the primary endpoint. In total 261 patients were randomized. The study was terminated early, since the statistical boundary of noninferiority has met the predefined stopping criteria. The 3-year OS rate was $59.1 \%$ (95\% CI $48.4-68.3 \%)$ in the bolus cisplatin group and $71.6 \%$ (95\% CI 61.7-79.4\%; $p=0.00272)$ in the weekly cisplatin arm and therefore noninferiority for weekly cisplatin demonstrated. Likewise, no difference was observed regarding RFS $(53.0 \%$; $95 \%$ CI $43.4-61.7 \%$ vs. $64.5 \%$; $95 \%$ CI $54.8-72.6 \%$ ). The cumulative cisplatin dose was above $200 \mathrm{mg} / \mathrm{m}^{2}$ in both arms. Most importantly, the side effect profile was different: Especially nephrotoxicity was higher in the bolus cisplatin group than in the experimental arm $(39.5 \%$ vs. $29.5 \%)$. The ESMO MCBS as rated by the author is $B$, i.e., relevant clinical benefit in the curative setting (noninferior OS, but improved toxicity compared to standard of care).

\section{R/M HNSCC}

Keynote 048: PFS 2 and CPS 1-19 subgroup analysis [6, 7]

In the pivotal phase III Keynote 048 trial in R/M HNSCC, first-line pembrolizumab (P) monotherapy and $\mathrm{P}$ plus chemotherapy $(\mathrm{P}+\mathrm{C})$ vs. Extreme SOC therapy (E) improved OS in PD-L1 combined positive score $(\mathrm{CPS}) \geq 20$ and $\mathrm{CPS} \geq 1$ populations with favorable safety. Data of further subgroup analyses of this study presented at the AACR and ASCO meetings support the use of this regimen as a new first-line standard for CPS-positive R/M HNSCC. Progression after the next line of therapy (PFS2), defined as time from randomization to objective tumor progression or death from any cause, was superior both in the $\mathrm{P}$ monotherapy arm in the CPS $\geq 20$ and $\geq 1$ (hazard ratio [HR] 0.64; 95\% CI $0.48-0.84$ and HR 0.80 ; 95\% CI $0.66-0.96$ ) subgroups and in the $\mathrm{P}+\mathrm{C}$ group (HR $0.63 ; 95 \% \mathrm{CI}$ $0.47-0.84$ and HR 0.66 ; $95 \%$ CI $0.54-0.80$ ) compared to E. The exploratory CPS 1-19 subgroup analysis suggested an improved OS for P+C vs E (HR 0.71; 95\% CI 0.54-0.94) and showed a HR of 0.86 (95\% CI 0.66-1.12) for $\mathrm{P}$ vs. E. The ESMO MCBS for $\mathrm{P}$ or $\mathrm{P}+\mathrm{C}$ as rated by the ESMO guideline committee is 4, i.e., relevant clinical benefit in the palliative setting.

\section{TPExtreme trial: quality of life and subsequent therapies [8]}

The TPExtreme study, which was published last year, investigated the efficacy and safety of TPEx (cisplatin/ docetaxel + cetuximab) vs. the SOC Extreme regimen. The study failed to demonstrate an OS advantage for TPEX, although the side effect profile was favorable. Further quality-of-life (QoL) data and analyses of subsequent therapies were reported this year. The global health status $(p=0.03)$, physical functioning $(p=0.02)$ and role functioning $(p=0.009)$ at week 18 was improved in the TPEx arm. More interesting, however, was the analysis of subsequent therapies: The median OS was longer in patients who received immunotherapy (19.5 months; 95\% CI 15.9-22.8) as a second-line treatment compared to those who received chemoor targeted therapy (16.2 months; 95\% CI 14.8-17.3). The difference was more pronounced in the TPEx arm than in the SOC arm.

\section{Nivolumab plus cetuximab [9]}

A single arm phase I/II study with $45 \mathrm{R} / \mathrm{M}$ HNSCC patients investigated the efficacy and safety of cetuximab plus nivolumab. Since prior therapy with an immune-checkpoint inhibitor was allowed, 51\% of the patients have been already exposed to immunotherapy. The overall response rate in the total population was $22.2 \%$. The 1-year PFS rate was $30 \%$ in the immunotherapy naïve group and $9 \%$ in the immunotherapy pretreated population suggesting that nivolumab plus cetuximab is more active in patients without prior immunotherapy expose. No safety issues were reported.

\section{Discussion}

Both the Senti-Mer and the E3311 studies demonstrate the feasibility end efficacy of de-escalation strategies in low or intermediate risk HNSCC. The 
results of the Senti-Mer study should be implemented into the clinical routine as soon as possible. Senti-Mer challenged the current paradigm that an elective neck dissection improves OS in an all comer population as demonstrated by D'Cruz et al. [10]. An upfront elective neck dissection can be avoided in cT1-T2, cN0, M0 OPC and OSCC patients if the sentinel lymph node biopsy was negative. This approach reduces the morbidity and the duration of the hospital stay without a negative impact on the outcome, although the initial intraoperative effort will be increased compared to an upfront elective neck dissection. The results of the E3311 study are promising but have to be confirmed in a larger phase III trial prior to its clinical applicability.

Since only $50 \%$ of locally advanced HNSCC patients can tolerate three cycles of high-dose cisplatin given in combination with radiotherapy, alternative treatment regimens are urgently needed. Unfortunately, there has been no randomized phase III study, which could show the equivalence of low dose cisplatin compared to the SOC. On the contrary, a recent study by Noronha et al. demonstrated that $30 \mathrm{mg} / \mathrm{m}^{2}$ weekly cisplatin is inferior to SOC bolus cisplatin in the adjuvant setting [11]. Therefore, the results of the JCOG1008 were eagerly awaited. How can the discrepancies between those two studies been interpreted? On the one hand, the cisplatin dose in the experimental arm was slightly higher in the JCOG1008 study than in the one of Noronha et al. and on the other hand the JCOG1008 experimental group achieved a cumulative cisplatin dose of $>200 \mathrm{mg} / \mathrm{m}^{2}$, which was not the case in the aforementioned study. Based on the JCOG1008 trial the $40 \mathrm{mg} / \mathrm{m}^{2}$ cisplatin dose might be considered as an alternative regimen, although some issues have to be noted: Since this study was solely conducted in Asia, the applicability for the rest of the world is unclear. Apart from that, it has to be stressed that the trial was not powered to show superiority of weekly cisplatin and one cannot conclude that this regimen is superior to the SOC, although the survival curves clearly separate.

As for the R/M setting, the data presented at the ASCO meeting confirm the current approach/ guidelines: $\mathrm{P} \pm \mathrm{C}$ is standard of care $\mathrm{CPS} \geq 1$ patients ( $\mathrm{P}$ is not approved in Europe for $\mathrm{CPS}=0$ patients). Based on the PFS 2 data presented at the ASCO meeting, it is tempting to speculate that first line immunotherapy sensitizes to subsequent therapies as well, although this hypothesis has to be confirmed in a prospective manner. In any case, the exploratory CPS 1-19 subgroup analysis suggest that the survival advantage observed for $\mathrm{P} \pm \mathrm{C}$ is not only driven by the CPS $\geq 20$ subgroup. Despite being not superior to the Extreme regimen, TPEx is an alternative especially in the $C P S=0$ setting due to the better safety and QoL profile. The optimal clinical approach after checkpoint inhibitor failure remains an unresolved question. Although combination strategies such as nivolumab plus cetuximab sound interesting, further (biomarker) studies are needed.

\section{Conclusion}

The HNSCC ASCO 2020 highlights which should be implemented into the clinical practice comprise (a) the Senti-Mer study, which established sentinel node biopsy as the SOC in T1-T2, N0, M0 OPC and OSCC and (b) and the JCOG1008 trial, which provided high-level evidence for postoperative weekly cisplatin plus radiotherapy as an alternative to highdose 3-weekly chemoradiation. Both studies showed an ESMO MCBS of $B$. Apart from that, additional subgroup analysis of the TPEx and Keynote 048 studies confirmed the current clinical practice.

Funding Open Access funding provided by Medical University of Vienna

Conflict of interest T. Fuereder is member of advisory boards and/or received honoraria from MSD, MERCK, Roche, Novartis, Amgen, Pfizer, BMS and Boehringer Ingelheim. Research funding by MSD and MERCK.

Open Access This article is licensed under a Creative Commons Attribution 4.0 International License, which permits use, sharing, adaptation, distribution and reproduction in any medium or format, as long as you give appropriate credit to the original author(s) and the source, provide a link to the Creative Commons licence, and indicate if changes were made. The images or other third party material in this article are included in the article's Creative Commons licence, unless indicated otherwise in a credit line to the material. If material is not included in the article's Creative Commons licence and your intended use is not permitted by statutory regulation or exceeds the permitted use, you will need to obtain permission directly from the copyright holder. To view a copy of this licence, visit http://creativecommons.org/licenses/by/4.0/.

\section{References}

1. Giuliani J, Bonetti A. Which grade is of clinical benefit in the randomised controlled trials? The example of 54th American Society of Clinical Oncology annual meeting, 2018. EurJCancer. 2018;104(1990):233-5.

2. Cherny NI, Dafni U, Bogaerts J, et al. ESMO-magnitude of clinical benefit scale version 1.1. Ann Oncol. 2017;28:2340-66.

3. Garrel R, Perriard F, Favier V, Richard F, Daures JP, Boutray MD. Equivalence randomized trial comparing treatment based on sentinel node biopsy versus neck dissection in operable T1-T2N0 oral and oropharyngeal cancer. JClin Oncol. 2020;38:6501.

4. Ferris RL, Flamand Y, Weinstein GS, et al. Transoral robotic surgical resection followed by randomization to low- or standard-dose IMRT in resectable p16+ locally advanced oropharynx cancer: a trial of the ECOG-ACRIN Cancer Research Group (E3311). JClin Oncol. 2020;38:6500.

5 . Kiyota N. Phase II/III trial of post-operative chemoradiotherapy comparing 3-weekly cisplatin with weekly cisplatin in high-risk patients with squamous cell carcinoma of head and neck (JCOG1008). ASCO. 2020.

6. Harrington KJ, Rischin D, Greil R, et al. KEYNOTE-048: progression after the next line of therapy following pem- 
brolizumab $(\mathrm{P})$ or $\mathrm{P}$ plus chemotherapy $(\mathrm{P}+\mathrm{C})$ vs EXTREME (E) as first-line $(1 \mathrm{~L})$ therapy for recurrent/metastatic $(\mathrm{R} / \mathrm{M})$ head and neck squamous cell carcinoma (HNSCC). J Clin Oncol. 2020;38:6505.

7. Burtness B. Efficacy of first-line pembrolizumab by PDL1 combined positive score $\langle 1,1-19$, and $\geq 20$ in recurrent and/or metastatic (R/M) head and neck squamous cell carcinoma (HNSCC): KEYNOTE-048 subgroup analysis AACR. 2020.

8. Guigay J, Fayette J, Mesia R, et al. TPExtreme randomized trial: Quality of Life (QoL) and survival according to secondline treatments in patients with recurrent/metastatic head and neck squamous cell carcinoma (R/M HNSCC). J Clin Oncol. 2020;38:6507.

9. Chung $\mathrm{CH}$, Bonomi MR, Steuer CE, et al. Concurrent cetuximab (CTX) and nivolumab (NIVO) in patients with recurrent and/or metastatic $(\mathrm{R} / \mathrm{M})$ head and neck squamous cell carcinoma (HNSCC): results of phase II study. J Clin Oncol. 2020;38:6515.
10. D'CruzAK, Vaish R, Kapre N, et al. Elective versus therapeutic neck dissection in node-negative oral cancer. N Engl J Med. 2015;373:521-9.

11. Noronha V, Joshi A, Patil VM, et al. Once-a-week versus once-every-3-weeks cisplatin chemoradiation for locally advanced head and neck cancer: a phase III randomized Noninferiority trial. JClin Oncol. 2018;36:1064-72.

Publisher's Note Springer Nature remains neutral with regard to jurisdictional claims in published maps and institutional affiliations.

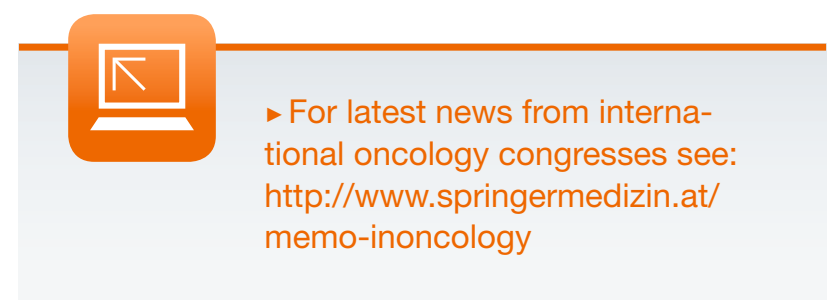

